\title{
Cardio-metabolic risk prediction should be superior to cardiovascular risk assessment in primary prevention of cardiovascular diseases
}

\author{
Hana Rosolova • Barbora Nussbaumerova
}

Received: 3 January 2011 / Accepted: 9 February 2011 /Published online: 5 March 2011

(C) European Association for Predictive, Preventive and Personalised Medicine 2011

\begin{abstract}
Cardiovascular atherosclerotic diseases represent the main cause of death in the developed and developing populations. Although major progress has been made in the management of the classical modifiable cardiovascular risk factors, unhealthy lifestyle conduces to an increasing prevalence of overweight, obesity, metabolic disorders, type 2 diabetes mellitus, premature atherosclerosis and cardiovascular diseases. That is why cardio-metabolic risk prediction should be superior in the primary prevention of atherosclerosis and cardiovascular diseases. Up-to-date primary preventive strategies according to the European Guidelines, especially the high risk strategy approach, are being implemented. Individual cardiovascular and better cardio-metabolic risk assessment represents the basic approach in the individualized primary prevention of cardiovascular diseases and type 2 diabetes mellitus. Cardiometabolic biomarkers, especially high sensitivity C-reactive protein, albuminuria, $\mathrm{N}$-terminal pro-brain natriuretic peptide, and imaging procedures (carotid intima-media thickness measured by ultrasound) could improve the prediction of cardiovascular diseases and type 2 diabetes beyond that using traditional risk factors.
\end{abstract}

Keywords Cardiovascular risk prediction · Primary prevention $\cdot$ Personalized cardio-metabolic risk $\cdot$ Imaging procedures $\cdot$ Cardio-metabolic biomarkers

\footnotetext{
H. Rosolova $(\varangle) \cdot$ B. Nussbaumerova

Centre of Preventive Cardiology of the 2nd Medical Department

University Hospital,

E. Benese 13,

30599 Pilsen, Czech Republic

e-mail: rosolova@fnplzen.cz
}

\section{Introduction}

A recent report from the World Health Organization (WHO) identified six risk factors as the leading global risk factors for death all over the world: high blood pressure, tobacco smoking, physical inactivity, overweight or obesity, and high cholesterol (C) levels. These factors contribute to a large proportion of the deaths from cardiovascular diseases (CVD), metabolic disorders, and cancer. These chronic noncommunicable diseases, the majority of which are preventable, are linked to the global lifestyle changes. The impact of long-term treatment and adverse effects on the quality of life, productivity, and disability take devastating tolls on the economic situation of individuals and societies alike [1].

Despite the decreasing rates of cardiovascular mortality in West European countries at the end of the 20th century, but in Poland and Czech Republic from the East European countries only, CVD have been the most common cause of mortality during the last 50 years. More than half of the European populations die from CVD, mostly from ischemic heart disease (Fig. 1) [2] That is why many activities of the European Society of Cardiology and, consequently, national societies of Cardiology are focused on preventive approaches to the most common cause of death, i.e., CVD. It was particularly the European Association for Cardiovascular Prevention and Rehabilitation (EACPR) [formerly the Working Group on Epidemiology and Prevention of Cardiovascular Diseases of the European Society of Cardiology], has consistently addressed this issue and developed the Joint Task Force Recommendations on CVD Prevention in cooperation with other medical societies regularly at a 4-year interval since 1994 [3]. 


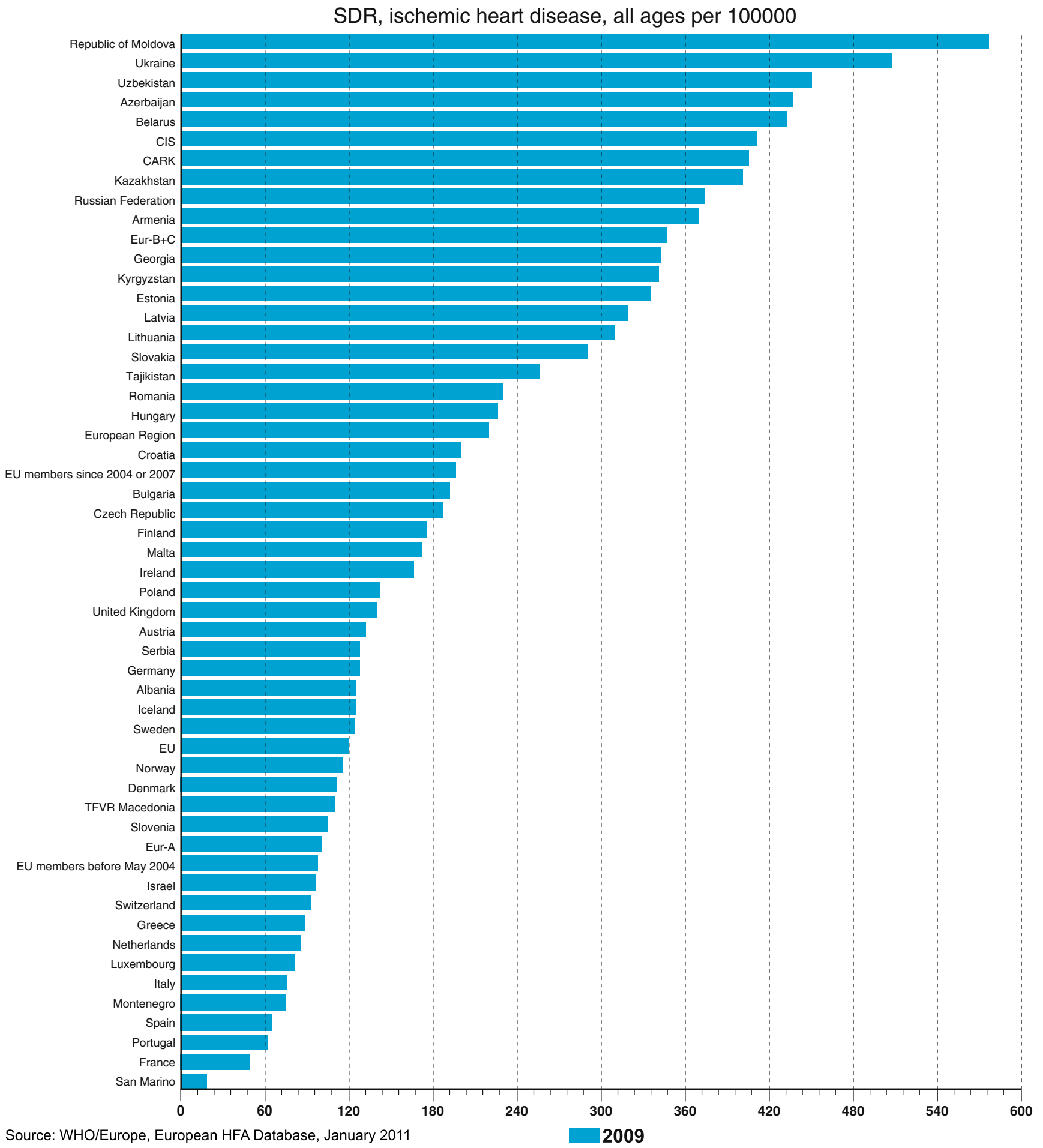

Fig. 1 Mortality of ischemic heart disease in European and other countries [2]. CARK - 5 central Asian republics including Kazakhstan, CIS - 12 countries of the "Commonwealth of Independent States", EU -

However, although impressive progress has been made in the management of the classical modifiable CVD risk factors (hypertension, hypercholesterolemia) in high risk individuals in CVD primary prevention, sedentary life-
European Union, Eur-A - Western European countries, Eur - B+C Middle and Eastern European countries

style and intake of high energy diet as well as tobacco smoking in a proportion of the population, represent typical up-to date lifestyle habits in the majority of European countries. These lifestyle habits conduce to the 


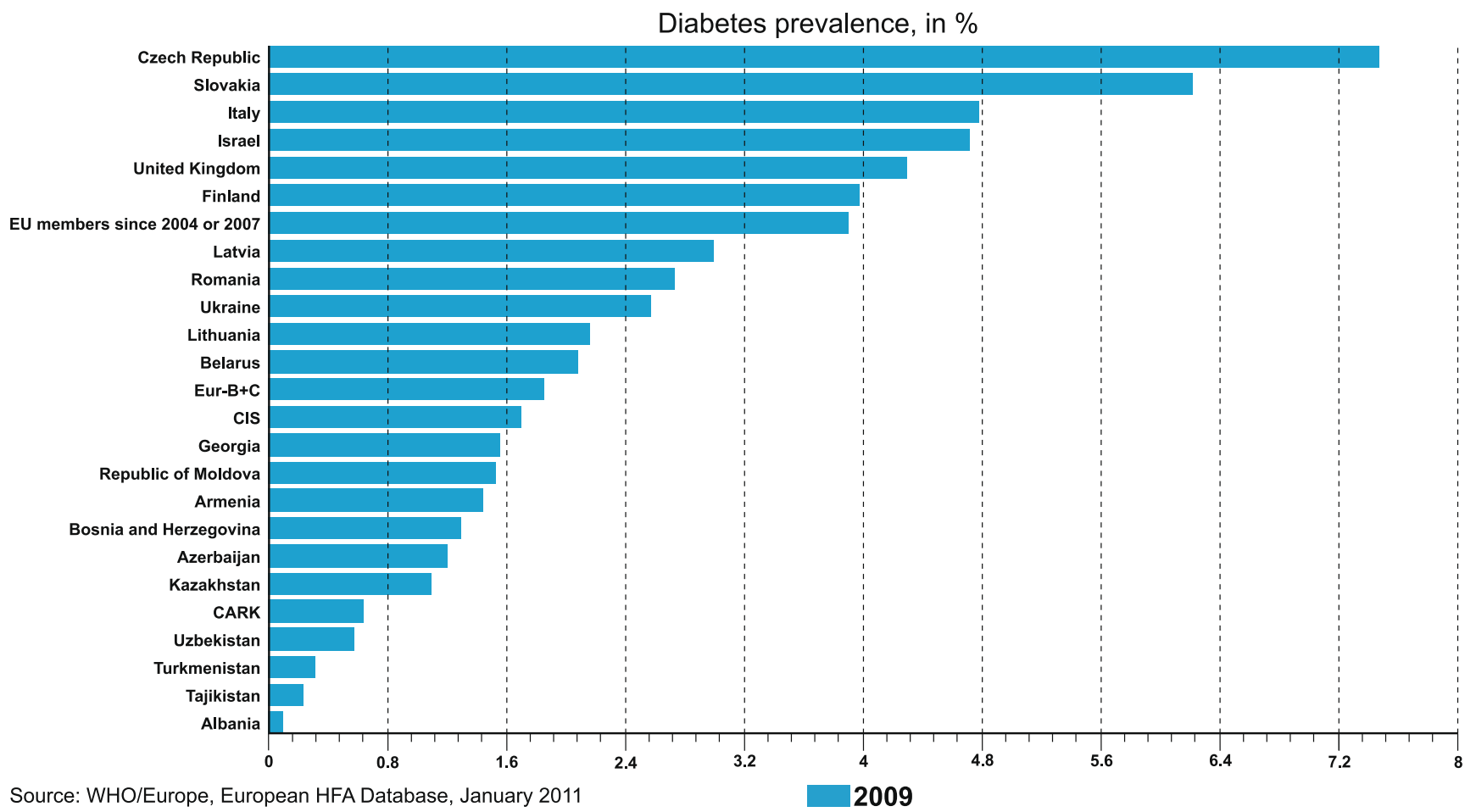

Fig. 2 Prevalence of type 2 diabetes mellitus in European and other countries [2], CARK - 5 central Asian republics including Kazakhstan, CIS 12 countries of the "Commonwealth of Independent States", Eur - B $+\mathrm{C}-$ Middle and Eastern European countries

increasing prevalence of overweight, obesity, metabolic disorders, and type 2 diabetes mellitus (T2DM) (Fig. 2) [2]. Many studies over the past years have shown that especially intra-abdominal fat accumulation is the link to a cluster of emerging metabolic risk factors that may increase the risk of T2DM and CVD. Metabolic abnormalities such as abdominal fatty tissue distribution, atherogenic dyslipidemia defined as elevated triglycerides (TG) concentration and reduced high-density lipoprotein (HDL)-C, impaired glucose homeostasis and elevated blood pressure cluster in subjects with insulin resistance (see below). These clustered risk factors were termed as "metabolic syndrome" (MS).

The relative risk (RR) for cardiovascular events in subjects with MS and even more so in diabetic subjects is significantly increased; 2 to 3 times in men and 3 to 4 times in women, respectively compared with subjects without MS and without T2DM, respectively $[4,5]$. The increased risk of CVD in T2DM subjects was only partly explained by hyperglycemia and only partly by other concomitant risk factors. The majority of patients with T2DM die from CVD [6]. Should the increasing trend of T2DM prevalence continue, the incidence of CVD will rise again! That is why preventive cardiologists have to pay utmost attention to the primary prevention of metabolic problems, and prevention of the growing incidence of $\mathrm{T} 2 \mathrm{DM}$ in particular.

\section{Cardiovascular diseases are multifactorial diseases}

In the early years of the preventive cardiology era, which come into being after the 2nd World War, atherosclerotic CVD were represented by ischemic heart disease only, to be joined by ischemic stroke, atherosclerotic stenosis of carotid arteries, peripheral occlusive arterial diseases, etc. later. Although atherosclerotic vascular diseases seem to be a more appropriate term for the above conditions, the term CVD has not been discarded in the majority of research papers. Epidemiologists and preventive cardiologists assessed and tested many causal risk factors for CVD, using cardiovascular epidemiological methods: increased plasma total $\mathrm{C}$ and/or low density lipoprotein (LDL)-C levels, tobacco smoking, increased blood pressure, age, and male sex. Causal risk factors have to met significant statistical criteria such as long-term, strong, specific, and time dependent association between the risk factor and disease. These criteria were tested in large epidemiological prospective and interventional trials $[7,8]$.

Cardiovascular diseases are multifactorial ones; various risk factors for CVD on the one hand, and genetic background on the other are both crucial preconditions for clinical manifestation of CVD. The genotype for the CVD phenotype is much more complicated; there are several variants of genes involved in lipid metabolism, hypertension, coagulation, endothelial function, etc. Individualized 
CVD prediction based on genetic testing is not available for clinical application yet because of several interactions between genes with each other and between genes and environmental factors. In everyday practice, a positive family history of CVD is useful for more precise CV risk assessment only; premature manifestation of any CVD in first-degree relatives (parents, son, daughter, brother, sister) in males under 55 years of age and in females under 65 years of age increases the risk of CVD about $50-70 \%$ as compared with subjects without a positive family history [3].

Since the late 1970s, new risk factors for CVD, so called emerging risk factors, including especially metabolic factors, have been identified. The first of these new metabolic risk factors was insulin resistance, i.e., a low sensitivity of cells and tissues to insulin-mediated glucose uptake. Together with compensatory hyperinsulinemia, abdominal distribution of adipose tissue, impaired glucose homeostasis (such as impaired fasting glucose, impaired glucose tolerance tested after a standard oral glucose load or T2DM), increased plasma TG levels and decreased protective HDL-C have been shown to be associated with insulin resistance. The fact that these metabolic abnormalities cluster in many individuals gave rise to the term "metabolic syndrome" (MS). Reaven was the first to refer to this accumulation of above mentioned dyslipidemia and high blood pressure as MS X [9]. It took many years to develop the definition of MS; the latest MS "harmonizing definition" is based on the presence of 3 or more of the five risk factors (Table 1). At present, MS is seen as a proatherosclerotic, pro-inflammatory and pro-coagulative status with a high risk for the premature development of atherosclerosis, CVD and T2DM [10]. All the established risk factors developing against the background of insulin resistance worsen with age and unhealthy lifestyle.

Subjects with MS and/or impaired glucose homeostasis are at an increased risk for premature atherosclerosis and, conversely, patients with established CVD are at high risk for metabolic disorders associated with insulin resistance [11]. The cardiovascular risk profile for CVD coincides with the risk profile for T2DM which is why the terms

Table 1 Definition of metabolic syndrome [10]

\begin{tabular}{ll}
\hline Waist circumference & $>88 \mathrm{~cm}$ in women \\
& $>102 \mathrm{~cm} \mathrm{in} \mathrm{men}$ \\
& $\geq 130 / 85 \mathrm{mmHg}$ \\
Blood pressure* & $\geq 1.7 \mathrm{mmol} / \mathrm{L}$ \\
Fasting triglycerides level* & $<1.3 \mathrm{mmol} / \mathrm{L}$ in women \\
HDL (high-density lipoprotein) & $<1.0 \mathrm{mmol} / \mathrm{L}$ in men \\
$\quad-$ cholesterol* & $\geq 5.6 \mathrm{mmol} / \mathrm{L}$ \\
Fasting glucose level* &
\end{tabular}

* or specific drug treatment cardio-metabolic risk profile and cardio-metabolic risk seem to be more appropriate and logical.

\section{Policy and strategies in cardiovascular disease prevention and assessment of individual cardiovascular risk in primary prevention}

Three strategies for the prevention of CVD are distinguished: population and high risk strategies in primary and secondary prevention among patients with manifest CVD. The population strategy is critical to reducing the overall incidence of CVD since it is designed to reduce risk factors at the population level. This population approach of recommendation and implementation of a healthy lifestyle, i.e., avoidance of tobacco smoking, healthy food choices, adequate physical activity and avoiding overweight and obesity, is not possible to achieve without national, and especially international community organizations and political institutions, e.g., national government, European Union (EU), WHO, etc. A new initiative, the European Heart Health Charter, was launched by the European Society of Cardiology, EU, and the European Heart Network in partnership with WHO (www.heartcharter.eu). The European Heart Health Charter advocates the development and implementation of comprehensive health strategies, measures and policies at European, national, regional and local levels that promote cardiovascular health and prevent CVD. The European Guidelines on cardiovascular disease prevention in clinical practice (the Fourth Task Force of the European Society of Cardiology and Other Societies on CVD Prevention in Clinical Practice) are intended to assist physicians and other health professionals to be successful in this endeavor, particularly in terms of employing effective preventive measures in day-to-day clinical practice [3].

The high risk strategy approach represents the physician's model in the primary prevention of CVD. In relatively healthy subjects without any manifest CVD, the risk chart should be used to estimate the global cardiovascular risk. The first risk chart, "the Framingham risk score", available since 1967 includes the following major (standard) risk factors: gender, age, tobacco smoking, systolic blood pressure and total $\mathrm{C}$ level. The absolute risk, i.e., the probability (\%) of non-fatal or fatal coronary events for the next 10 years, was estimated in each person in the primary prevention of CVD according to the above risk factors. There were several problems with this chart; because the data were derived from the American population, and obtained from the not very large Framingham study, only coronary events could be assessed. In 2003, a new risk chart was introduced in the European Guidelines on CVD Prevention: "SCORE_European High (Low) Risk Chart" based on the data from 12 European CVD mortality 
population studies including more than 200,000 men and women [12]; regrettably, non-fatal events were not evaluated in these studies. Two types of Score risk charts are currently available, one estimating cardiovascular risk based on standard risk factors, i.e., gender, age, smoking, systolic blood pressure and plasma total $\mathrm{C}$ level, and the other for subjects with low HDL-C levels (below $1.3 \mathrm{mmol} / \mathrm{L}$ in women and below $1.0 \mathrm{mmol} / \mathrm{L}$ in men) on the basis of the above standard risk factors, with the only difference being that total C level is replaced by total C/HDL-C levels. More recently, some European countries including the Czech Republic, developed their own Score risk charts based on the fatal events reported from their own population studies [13]. An example of the SCORE Risk Chart for the high risk European countries is shown in Fig. 3.

Assessment of the absolute global risk for CVD in asymptomatic persons reflects the personalized approach of the prediction and prevention of CVD, with healthcare differentiated according to the actual individualized risk score. The absolute risk may be higher than indicated in the chart; e.g., in sedentary or obese subjects, especially those with abdominal obesity, those with a strong family history of premature CVD, the socially deprived, those with low HDL-C or high TG, and in asymptomatic subjects with evidence of pre-clinical atherosclerosis, e.g., a reduced ankle-brachial index, or documented by imaging techniques such as carotid ultrasound or computed tomography, etc. A very intensive healthcare concerning lifestyle improvement and pharmacological treatment of risk factors is necessary in high risk subjects with a $5 \%$ or higher probability of any fatal CVD over the next 10 years. Subjects with a low global CV risk have to pursue a healthy lifestyle to maintain a low CV risk. It is necessary to look for other metabolic risk factors, especially those not included in the Risk Score tables yet. Subjects with intermediate CV risk (3-5\%) make up the majority of the general population; however, while having average risk factors levels, they develop a high number of $\mathrm{CV}$ events.

There is evidence to suggest that the current risk assessment algorithm using traditional risk factors may not estimate the global CV risk accurately, especially in patients with insulin resistance and accompanying risk factors such as abdominal obesity, atherogenic dyslipidemia (high TG and reduced HDL-C levels), impaired glucose homeostasis, etc. Many studies have compared the performance of the classical tools of $\mathrm{CV}$ risk evaluation (Framingham risk score, SCORE Risk Chart) and MS in CVD prediction. The presence of MS usually does not impact the risk in subjects at high risk, whereas the presence of MS in subjects at low or intermediate risk significantly increases the probability of developing CVD, making it imperative to improve their global cardiometabolic risk assessment.
Fig. 3 SCORE - European Low Risk Chart [3]

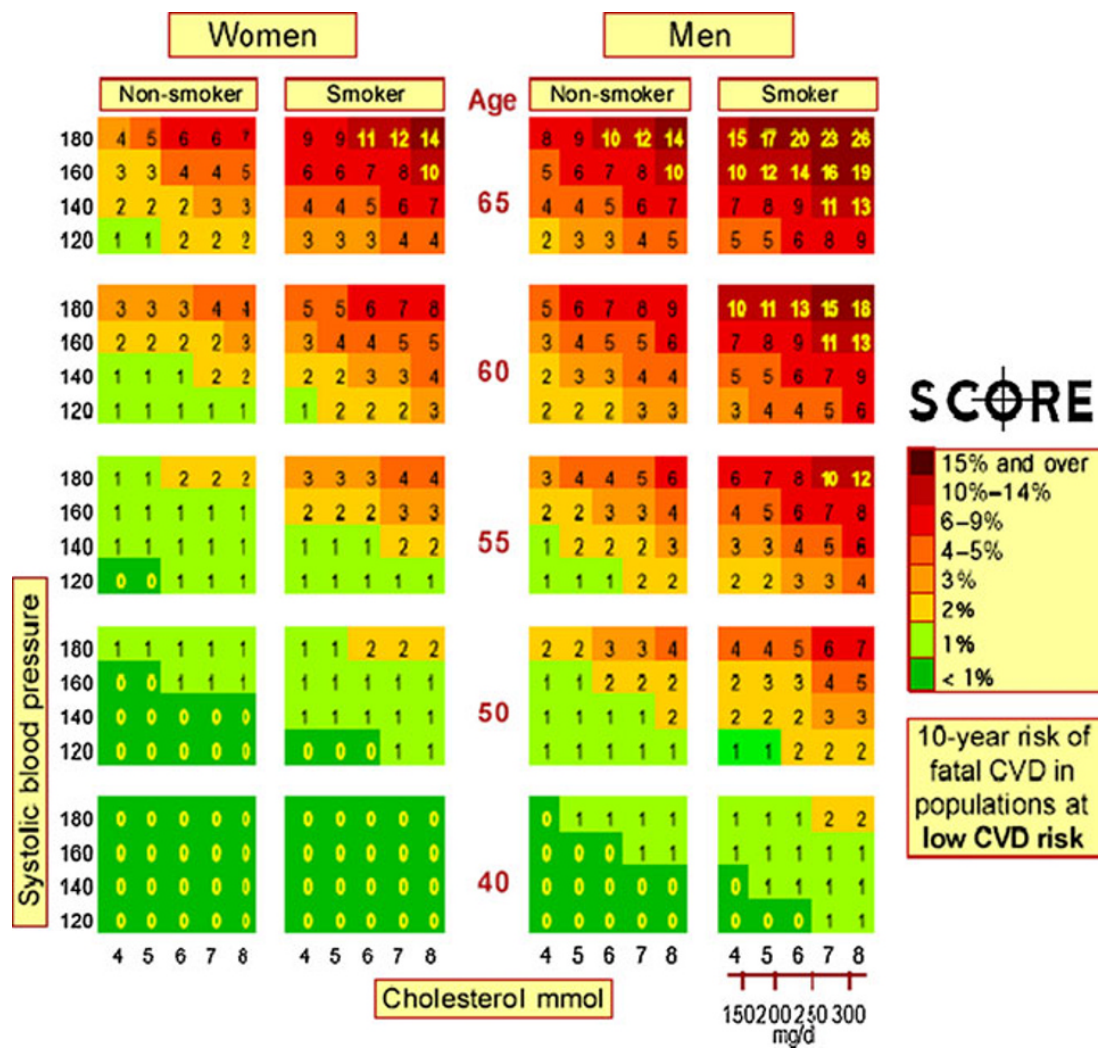




\section{Risk factors associated with insulin resistance, biomarkers and imaging procedures could improve cardio-metabolic risk assessment}

Metabolic syndrome and type 2 diabetes mellitus

It should be noted that the five risk factors of MS summarized in Table 1 were proposed by expert consensus while not tested for their ability to allow for the identification of individuals both with MS and the related increase in CVD risk [14]. Clinicians have often been confused by the conceptual definition of MS with the proposed criteria. The risk factors of MS are surrogate variables simply reached in clinical practice to identify high risk subjects. The dichotomously divided five risk factors should help clinicians unable to measure insulin resistance using very difficult and time consuming methods such as the euglycemic hyperinsulinemic clamp or the insulin suppression test, accurately measure visceral adiposity by expensive computed tomography or magnetic resonance imaging, etc.

The concept of MS, a syndrome of abnormalities linked to insulin resistance, was introduced by Reaven in California in 1988 [9]. He proposed that impaired insulin action and compensatory hyperinsulinemia were both part of the MS cluster, together with elevated blood pressure, hypertriglyceridemia and low HDL-C. As Reaven and his colleague found insulin resistance, as assessed by the insulin suppression test, also in lean subjects, their definition of MS did not include obesity. Much earlier, Vague drew attention to the importance of abdominal adiposity (upper body adiposity, android or male type obesity are synonymous) as a risk factor for CVD and T2DM [15]. The use of very sophisticated imaging techniques, e.g., computed tomography or magnetic resonance imaging, to measure abdominal fat accumulation, has shown that independently of the presence or absence of clinical obesity defined as $\mathrm{BMI} \geq 30 \mathrm{~kg} / \mathrm{m}^{2}$, an increase of intraabdominal or visceral adipose tissue correlates significantly with the occurrence of MS and other known CV risk factors.

\section{Waist circumference}

Waist circumference reflects abdominal distribution of adipose tissue and is a most simple non-time consuming method for everyday practice. It is recommended to measure waist circumference in the upright position, at mid-distance between the chest and the spina illiaca anterior superior, when the subject has exhaled. Waist circumference values $>94$ (or $>102)$ cm in males and $>80$ (or $>88$ ) $\mathrm{cm}$ in females are significantly associated with overweight (or abdominal obesity) and high $\mathrm{CV}$ and metabolic risk.
However, the above values are not enough for the clinical identification of high risk subjects for CVD and T2DM. Several groups have reported that an increase in waist circumference and fasting TG levels (described as the hypertriglyceridemic waist) may be relevant and readily screened measures to identify subjects at high risk of MS [16]. Reduction of waist circumference by diet or pharmacological treatment in obese subjects leads to a reduction of intra-abdominal fat while improving insulin resistance and reducing the CVD and metabolic risk as shown by the Quebec Cardiovascular Study [17].

\section{Blood pressure}

Casual blood pressure (BP) is one of the traditional cardiovascular risk factors, a $\mathrm{BP} \geq 130 / 80 \mathrm{mmHg}$, including high normal $\mathrm{BP}=130-139 / 80-89 \mathrm{mmHg}$, is associated with a higher $\mathrm{CV}$ and metabolic risk than a $\mathrm{BP}<130 / 80 \mathrm{mmHg}$ [18]. This association is known in primary preventive studies of CVD only. In secondary CVD prevention, and especially in patients with heart failure, a BP below 130/80 is consistent with a worse prognosis compared with subjects treated to achieve have BP below 140/90 but not less than $130 / 80 \mathrm{mmHg}$ [19]. It is very well known from interventional studies that a healthy lifestyle, characterized especially by a rational diet with reduced salt intake, regular physical activity and non-smoking, is advisable in subjects with high normal BP. Antihypertensive drugs are indicated in subjects with repeatedly high $\mathrm{BP} \geq 140 / 90 \mathrm{mmHg}$ and high global CVD risk. Numerous pharmacological trials have clearly demonstrated that personalized treatment of hypertension is needed. In hypertensive patients with metabolic disorders such as MS or T2DM, inhibitors of the renin-angiotensin system (RAS) are the first choice drugs (ACE inhibitors or angiotensin II receptors blockers). These drugs do not only reduce BP but also improve insulin resistance while not worsening lipid metabolism. Antihypertensive treatment by RAS inhibitors reduces $\mathrm{CV}$ risk and, also, the risk for development of T2DM, which is the most important effect in subjects with MS. Combination therapy is very often needed to achieve a BP $<140$ / $90 \mathrm{mmHg}$ [3]. Calcium channel blockers, metabolically neutral indapamid/metapamid or centrally acting antihypertensives stimulating imidazoline receptors in the central nervous system are suitable for combination with RAS inhibitors in patients with MS or T2DM.

\section{Dyslipidemia}

Low density lipoprotein cholesterol (LDL-C) is considered the main risk factor for atherosclerosis and CVD; LDL particles are involved in the atherosclerotic process, and the higher LDL-C, the higher CV risk. Recommended levels of 
total and LDL-C are below $5 \mathrm{mmol} / \mathrm{L}$ and below $3 \mathrm{mmol} / \mathrm{L}$, respectively in subjects in primary CVD prevention. On the other hand, more reduced levels of total and LDL-C (the higher the CVD risk, the lower LDL-C) are recommended in patients with manifest CVD or with T2DM. Reducing LDL-C levels by statin therapy will lower the incidence of CVD by up to one third. Although impressive, these results also raise the question of how to decrease the residual relative $70 \%$ risk of CVD events that still occur? Atherogenic dyslipidemia is one of the targets of current research, with the Residual Risk Reduction Initiative (R3i), a non-profit organization, established to address this important issue [20].

Some clinical and epidemiological studies showed recently that high TG levels are also associated with higher $\mathrm{CV}$ risk than TG levels below $1.7 \mathrm{mmol} / \mathrm{L}$. Triglyceride levels are determined as part of standard biochemical venous blood sample screening. In fact, TGs are the most problematic particles in terms of $\mathrm{CV}$ risk prediction and prevention of atherosclerosis. Triglyceride-rich particles are most heterogeneous; they are known to be present in chylomicrons, VLDL, IDL and, in smaller amounts in LDL, which is why TG levels in multifactorial studies were not always assessed as an independent CVD risk factor. This explains why apolipoproteins B (part of atherogenic lipid particles LDL, VLDL, IDL) and apolipoproteins A (part of the anti-atherogenic particles HDL), and their ratio predict CVD risk more precisely as showed in, e.g., the Interheart study [5]. Triglyceride levels above $1.7 \mathrm{mmol} / \mathrm{L}$ are known to be associated with the presence of small dense LDL particles which are very atherogenic, susceptible to oxidation and glycation, and resistant to the LDL receptors. All these features explain their high atherogenic activity. Elevated TG levels, small dense LDL particles and low HDL-C are called the lipid triad characterized by atherogenic dyslipidemia that often occurs in subjects with MS or T2DM [21].

Plasma HDL-C is considered a cardioprotective lipid particle independent of other major $\mathrm{CV}$ risk factors. It has been long known (since the 1950s) that the lower the HDL$\mathrm{C}$ levels, the higher the CV risk in both genders [22]. The outcomes of clinical trials evaluating HDL-C-raising therapies lack the strength and consistency of the statin trials data. Torcetrapib therapy in subjects with reduced HDL-C levels was stopped and the drug was withdrawn from the market because of a significant increase in CVD mortality in addition to a significant rise in HDL-C.

Extensive evidence shows that elevated TG and low HDL-C are both predictors for CVD independent of LDLC. Non-fasting TG levels, measured $2-4 \mathrm{~h}$ postprandially, may be of even greater relevance to CVD risk since atherogenic lipoprotein remnants secreted by the liver and intestine postprandially circulate at higher concentrations than in the fasting state, as supported by evidence from clinical studies [23].

Furthermore, the total C/HDL-C ratio also needs to be taken into account, as shown by the Prospective Cardiovascular Münster (PROCAM) study. In this study, one in seven subjects with a combination of a high total C/HDL-C ratio $(>5.0)$, low HDL-C $(<0.90 \mathrm{mmol} / \mathrm{L})$ and elevated TG $(>2.26 \mathrm{mmol} / \mathrm{L})$ experienced myocardial infarction [24]. Many other atherogenic indexes are used for atherosclerosis prediction, e.g., the ratio of fasting TG to HDL-C has been shown to be a good marker of insulin resistance [25].

Some years ago, Dobiasova and Frohlich proposed an atherogenic index of plasma levels of TG and HDL-C: $\mathrm{AIP}=\log$ [TG/HDL-C] [26]. Logarithmic transformation of the molar concentrations of TG and HDL-C is closely related to the sizes of HDL-C, LDL-C and VLDL-C particles, which are now considered to be new-generation indicators of $\mathrm{CV}$ risk and define the atherogenic genotype of plasma more precisely than classical biochemical markers such as LDL-C, HDL-C, total-C, apolipoprotein $\mathrm{B}$ or A, etc. The authors have improved that AIP can be used for monitoring the actual lipoprotein profile and predicting $\mathrm{CV}$ risk. Increased TG levels are associated with increased levels of atherogenic lipid particles (in particular LDL and VLDL) and increased rates of transport of cholesterol esters from HDL particles to lipoproteincontaining Apo-B. While the levels of TG alone are not an independent risk marker, they do serve as a regulator of lipoprotein interactions. Some studies have shown that AIP may be an important tool for analyzing the results of clinical trials [27].

Regarding T2DM patients, only data from the ACCORD trial are available; only in those diabetic patients with atherogenic dyslipidemia defined as $\mathrm{TG} \geq 2.3 \mathrm{mmol} / \mathrm{L}$ with HDL-C $<0.9 \mathrm{mmol} / \mathrm{L}$ did combination hypolipidemic therapy with simvastatin plus fenofibrate reduce CVD risk as compared with T2DM patients without this atherogenic dyslipidemia [28]. No data are available on the treatment of atherogenic dyslipidemia in subjects without T2DM, e.g., in subjects with MS. Preventive cardiologists suggest that more studies and more pharmacological trials for atherogenic dyslipidemia treatment are warranted to obtain a larger body of evidence.

\section{Glucose}

Elevated fasting plasma glucose level is also considered an independent risk factor for CVD; it is especially the postprandial glucose level that is better predictive for $\mathrm{CV}$ risk and risk for T2DM.

A fasting glucose level of more than 5.6 and less than $7.0 \mathrm{mmol} / \mathrm{L}$ is considered impaired fasting glucose and only a proportion of these subjects also have impaired glucose 
tolerance as assessed by oral glucose tolerance test (oGTT). If the glucose level at $2 \mathrm{~h}$ after oral $75 \mathrm{~g}$ poor glucose intake is found to be between 7.8 and $11 \mathrm{mmol} / \mathrm{L}$; impaired fasting glucose and impaired glucose tolerance are often called "prediabetes". Levels of $11 \mathrm{mmol} / \mathrm{L}$ and higher at $2 \mathrm{~h}$ of oGTT are considered T2DM. Pharmacological reduction of hyperglycemia is recommended in diabetic patients (repeatedly increased fasting plasma glucose of $7 \mathrm{mmol} / \mathrm{L}$ or oGTT, see above) only and metformin is drug of the first choice. A reduction of CVD morbidity and mortality was documented in the UKPDS study (United Kingdom Prospective Diabetes Study) in obese patients with newly diagnosed T2DM. Other studies have found many discrepancies in terms of antidiabetic pharmacological treatment and CVD risk. Whilst some old oral antidiabetic drugs increased CVD risk, the new ones are more efficient not only for hyperglycemia but, also, in the prevention of CVD. As shown in the most recent pharmacological trials, glucose control in patients with diabetes has to be individualized. Intensive antidiabetic therapy is recommended in newly diagnosed $\mathrm{T} 2 \mathrm{DM}$ as against T2DM of long duration. Intensive therapy with glucose-lowering drugs produces a number of hypoglycemic events and increases the $\mathrm{CV}$ risk in patients with long duration of T2DM [29]. All glucose disorders described above including T2DM may be present in subjects with MS.

Should fasting or postprandial glucose levels or glycosylated hemoglobin $\left(\mathrm{HbA}_{1 \mathrm{C}}\right)$ levels be included into the CVD risk assessment to improve the accuracy of cardiometabolic risk assessment? The only CVD risk score, which is the only one of its kind including impaired fasting glucose and impaired glucose tolerance in the risk function determination was developed by the DECODE group (Diabetes Epidemiology: Collaborative analysis Of Diagnostic criteria in Europe) [30]. The Finnish Diabetes Risk Score (FINDRISC) predicts the 10-year risk for developing T2DM with an $85 \%$ accuracy. It is based on a questionnaire suitable for the general population to identify subjects at high risk for T2DM. Its items include age, BMI, waist circumference, physical activity, diet, information about hypertension, glucose level from the past and family history of diabetes; sum of the points related to the specific answer shows the total score of risk for developing T2DM within 10 years. In addition, FINDRISC predicts the incidence of myocardial infarction and stroke [31].

High-sensitivity C-reactive protein, albuminuria, and brain natriuretic peptide as biomarkers

Various biomarkers have been investigated to improve the prediction of individual cardio-metabolic risk in subjects from the general population over the last two decades.
Many of these markers associate with inflammation which plays an important role in all stages of the atherosclerotic process.

C-reactive protein evaluated by the high sensitivity method (hs-CRP)

High sensitivity CRP is an acute phase protein belonging to the family of proteins known as pentraxins; it is synthesized primarily by hepatocytes but, also, by macrophages and smooth muscle cells. It is a marker of systemic inflammation. Recently, our research group found in patients with $\mathrm{T} 2 \mathrm{DM}$ that $\mathrm{hs}-\mathrm{CRP} \geq 3 \mathrm{mg} / \mathrm{L}$ is a common feature, particularly in diabetics with abdominal obesity who meet the criteria of MS. Waist circumference (but not body mass index) appeared to be a major variable associated with increased hs-CRP independent of age and sex in our study [32]. High sensitivity CRP has been shown to be an independent predictive factor for CVD and, also, for MS [33]. The largest meta-analysis to date has included individual records of 160,309 people without a history of vascular disease from 54 long-term prospective studies; CRP levels were linearly associated with several conventional risk factors and inflammatory markers, and nearly linearly with the risk of ischemic vascular disease and nonvascular mortality. Risk ratios (RRs) for coronary heart disease per 1-SD higher log CRP levels (three times higher) were $1.63(95 \%$ CI $1.51-1.76)$ when initially adjusted for age and sex only, and $1.37(1.27-1.48)$ when further adjusted for conventional risk factors; 1.44 (1.32-1.57), and $1.27(1.15-1.40)$ for ischemic stroke; $1.71(1.53-1.91)$, and $1.55(1.37-1.76)$ for vascular mortality. The authors summarized in the meta-analysis that hs-CRP levels showed continuous associations with the risk of coronary heart disease, ischemic stroke, and vascular mortality [34].

C-reactive protein, as assessed by the high sensitivity method, seems to be actually independent and may be a causal risk factor for CVD and T2DM in the near future. Its levels are affected by genetic polymorphisms and environmental factors (especially by dietary components). It is known from many pharmacological studies that statins or fibrates can reduce hs-CRP levels in some patients. Future studies focusing on the effects and interactions of genetic variation on the hs-CRP response to dietary and other behavior modifications as well as drug treatment could have important implications for the development of more personalized preventive and therapeutic approaches to reduce cardio-metabolic risk.

\section{Albuminuria $(A U)$}

Albuminuria is defined as excretion of albumin in the urine; microalbuminuria $30-300 \mathrm{mg} / 24 \mathrm{~h}$, macroalbuminuria $>$ 
$300 \mathrm{mg} / 24 \mathrm{~h}$, and/or reduced glomerular filtration rate $(\mathrm{GFR})<60 \mathrm{~mL} / \mathrm{min}$ were both recognized as significant risk factors for CVD and glucose metabolism disorders such as T2DM [3]. Albuminuria reflects injury to the endothelium, not only in glomerular endothelium but, also, the endothelium of other arteries of the body. Albuminuria was associated not only with impaired renal function but, also, with CV risk, metabolic risk, and total mortality [35]. A more precious method is the expression of $\mathrm{AU}$ as the creatine/albumin ratio (UACR, in $\mathrm{mg} / \mathrm{mmol}$ ). A simple semiquantitative method using the dipstick test is recommended in all patients with arterial hypertension and/or diabetes. Albuminuria is reduced by a healthy lifestyle, especially after giving up smoking or after treatment of hypertension (RAS inhibitors are very effective in reducing AU independently of systematic blood pressure reduction) or hyperglycemia [36, 37].

Given the increasing prevalence of T2DM and renal diseases, widespread use of the dipstick test in the general population is still under debate. However, the AU dipstick test is an easy and inexpensive test for the identification of subjects with cardio-metabolic and renal risk; a positive finding of AU has to be related to other specific risk factors for the above diseases.

\section{$N$-terminal pro-brain natriuretic peptide (Nt-proBNP)}

Brain natriuretic peptide is considered a very useful clinical marker and predictor for diagnosis and prognosis, respectively, in patients with heart failure in clinical practice [38]. Our research group has shown recently that the elevation of Nt-proBNP increased more than three times the RR for total mortality in asymptomatic patients with stable coronary heart disease [39].

There are less data concerning Nt-proBNP in the prediction of $\mathrm{CV}$ and metabolic risk in primary prevention. Our colleagues from the Research Center for Prevention and Health from Glostrup University Hospital in Denmark showed, in a general population sample, that hs-CRP was primarily related to metabolic $\mathrm{CV}$ risk factors whereas $\mathrm{Nt}$ pro-BNP was primarily related to hemodynamic risk factors and urinary albumin/creatinine ratio (UACR) related to both hemodynamic $\mathrm{CV}$ risk and metabolic risk factors [40].

Only a small number of biomarkers was analyzed comprehensively with traditional $\mathrm{CV}$ risk factors and/or known markers of vascular damage. Many of these biomarkers were significantly associated with cardiometabolic risk in bifactorial analysis, many of them lost their significance in multifactorial analysis, i.e., after adjustment for the standard risk factors having a stronger statistical power than the emerging risk factors. These results from large prospective randomized epidemiological studies using standard sophisticated statistical methods are considered to be the most relevant for clinical practice, so-called evidence-based medicine data. It is the epidemiological approach, used also in interventional studies, which is the most important for the evaluation of risk factor causality. On the other hand, there are many individual combinations of known risk factors, emerging risk factors, etc., and our ultimate goal is to identify some markers or group of markers to more precisely determining absolute personalized risk for atherosclerosis and/or metabolic disorders and then institute personalized preventive measures early enough to stop the development of the disease.

Imaging techniques

A host of new imaging techniques have come into use over the last 10 years; these include computed tomography (CT) angiography, calcium score assessment by CT, magnetic resonance imaging, etc. Most of them are not suitable for primary preventive screening in the general population.

\section{Carotid intima-media thickness}

Ultrasound carotid intima-media thickness (CIMT) measurement is a non-invasive, reproducible, inexpensive, and radiation-free screening test. Recent data have improved our understanding of the application of CIMT as a screening tool for CVD. Carotid intima-media thickness measurement may move an individual into a higher- or lower-risk category, allowing for appropriate institution of preventive strategies. The predictive value is better for stroke than for cardiac events. Nevertheless, coronary heart disease prediction by CIMT is inferior to that by ultrasound carotid plaque assessment as the plaque may be more representative of atherosclerosis than CIMT. In the Finnish population-based Health 2000 Survey, CIMT was used as a marker of subclinical atherosclerosis. In multivariate models, MS was an independent determinant of CIMT in both sexes ( $p \leq 0.001$ for both). When MS was included into the regression models along with its components, it was an independent determinant of CIMT in women but not in men. After dividing the population into risk categories according to the Framingham risk score and the presence of MS, the Framingham risk score predominantly determined CIMT regardless of the presence of MS in men. However, in women, CIMT was significantly higher in subjects with MS than in those without it, independently of the Framingham risk score. In conclusion, $\mathrm{MS}$ is an independent determinant of CIMT in both sexes. In women, but not in men, MS is associated with CIMT independently of its components and provides additional information on a person's risk for early atherosclerosis beyond the Framingham risk score in women, but not in men [41]. 


\section{Coronary calcium}

Coronary calcium in the coronary arteries wall is a marker of the presence of atherosclerosis. The assessment is most often performed using multislice computed tomography, with the calcifications quantified by the Agatston score (CAC). CAC can not be interpreted mechanically; it has the strongest association with age and male gender [42]. Subjects with MS and T2DM have been shown to have more likely higher CAC than the general population [43, 44]. The prognostic value of CAC is shown in the Table 2. The predictive CAC value was shown, e.g., in a follow-up of 11, 000 patients undergoing a screening medical examination including the CAC score in 1995-2000. During a mean follow-up period of 3.5 years in asymptomatic men and women, CVD events (non-fatal myocardial infarction and CVD-related deaths) were higher with a CAC score $>400$ [45]. A negative CAC shows a strong negative predictive value for future CVD events and the presence of plaques is uncommon in these subjects [46]. In contrast to hs-CRP or CIMT, there is actually no possibility to significantly modulate CAC by treatment of standard risk factors, e.g., by the statin therapy of hyperlipidemia [47].

At present, routine quantification of CAC progression cannot be recommended in clinical practice because of the lack of clinical data and protocols [48]. There is no significant correlation between CAC and myocardial stress testing [49], and stress testing or coronary angiography in asymptomatic subjects with high CAC is not recommended. In conclusion, CAC is considered a marker of stable atherosclerotic disease with an important predictive value for CVD. The major limitations of CAC are its cost and radiation.

A very interesting question remains to be answered. What is the background of the association between MS, CIMT, and CAC? The Atherosclerosis Risk in Communities (ARIC) study could explain a part of the above question. A sample of 7260 non-diabetic Caucasian individuals with IMT measurement and relevant genotyping was formed and a fasting glucose genetic risk score developed according to the strength of genes influencing the fasting glucose. This genetic score was significantly

Table 2 Agatston score and cardiovascular risk [48]

\begin{tabular}{ll}
\hline Agatston score & Cardiovascular risk \\
\hline 0 & Minimal \\
$1-10$ & Low \\
$11-99$ & Intermediate \\
$100-400$ & High \\
over 400 & Very high \\
\hline
\end{tabular}

associated $(p=0.009)$ with mean CIMT. The significant association of the fasting glucose genetic risk score with IMT suggests a possible a causal association of elevated fasting glucose with atherosclerosis (although it may be that these loci affect IMT through non-glucose pathways) [50].

Another genetic survey explored a genetic variant of T2DM strongly associated with CAC. In contrast, variants near insulin-like growth factor-binding protein, transcription factors, several transporters were clearly associated with diabetes; no evidence for an association with CAC was observable. This differential association pattern underlines the potential of endophenotypes such as CAC, to extend the scope of disease outcome [51]. In conclusion, it is clear that the relationships between the findings of imaging and laboratory methods are based on a strong genetic background.

Both non-invasive imaging methods, CAC and CIMT, are significant predictors of CVD and are significantly associated with MS. Although CIMT is less expensive with no radiation, $\mathrm{CAC}$ shows a better prognostic value.

\section{Conclusion and main messages}

Recent clinical studies have shown that the traditional CV risk prediction score system needs further improvement, because the majority of $\mathrm{CV}$ events occurs in intermediateor partly in low-risk groups of the population. Many metabolic risk factors, markers or predictors have been identified during the last two decades. It is especially because of the risk factor clustering that MS needs to be focused on in personalized prediction and early prevention of premature atherosclerosis. The increasing prevalence of T2DM is a consequence of an unhealthy lifestyle, particularly sedentary habits and high energy food intake on one hand, and late identification and start of treatment of cardiometabolic risk factors on the other.

New biomarkers, hs-CRP, abuminuria and Nt-proBNP are associated with the standard CV risk factors (age, sex, tobacco smoking, high blood pressure and high plasma cholesterol), and seem to be useful predictors in cardiometabolic risk assessment. However, sophisticated imaging techniques such as carotid intima-media thickness, presence of atherosclerotic plaques or high coronary artery CAC, could improve cardio-metabolic risk prediction; their use in primary prevention is limited, because some of them are too expensive, time-consuming, and invasive in radiation.

The efficacy of preventive tools, especially of CVD and T2DM primary prevention, depends on the personalized approach to subjects at high cardio-metabolic risk in the general population. It is particularly general practitioners who are responsible for preventive screening and identification of subjects at high cardio-metabolic risk. Well- 
educated general practitioners, with the help of all other specialists, have to implement preventive measures and start interventions early and rationally according to latest concepts in evidence-based medicine.

The many barriers to better CVD and T2DM prevention are still evident. In fact, they reflect the difficulty in making accurate CVD risk estimation in the clinical setting, particularly in the intermediate-risk subjects. The limitations of CVD risk prediction tools such as underutilization of the current CVD risk calculators, especially in primary care, are obvious. An additional barrier is the failure to effectively implement guideline recommendations on risk factors management and CVD prevention.

\section{References}

1. Global health risks: mortality and burden of disease attributable to selected major risks. Geneva: WHO, Dec 2009. Assessed at: http://www.who.int/healthinfo/global_burden_disease/Global HealthRisks_report_full.pdf

2. http://data.euro.who.int/hfadb

3. Graham I, Atar D, Borch-Johnsen K, et al. Task Force Members: European guidelines on cardiovascular disease prevention in clinical practice: executive summary. Fourth Joint Task Force of the European Society of Cardiology and other societies on cardiovascular disease prevention in clinical practice (constituted by representatives of nine societies and by invited experts). Eur J Cardiovasc Prev Rehabil. 2007;14(Supp12):E1-40.

4. Laakso M. Hyperglycaemia and cardiovascular disease in type 2 diabetes. Diabetes. 1999;48:937-42.

5. Yusuf S, Hawken S, Ounpuu S, et al. Effect of potentially modifiable risk factors associated with myocardial infarction in 52 countries (the INTERHEART study): a case-control study. Lancet. 2004;364:937-52.

6. Morrish NJ, Stevens LK, Head J, Fuller JH, Jarrett RJ, Keen H. A prospective study of mortality among middle-aged diabetic patients (the London Cohort of the WHO Multinational Study of Vascular Disease in Diabetics) II: Associated risk factors. Diabetologia. 1990;33:542-8.

7. Research Group of MRFIT. Coronary heart disease death, nonfatal acute myocardial infarction and other clinical outcomes in the Multiple Risk Factor Intervention Trial. Multiple Risk Factor Intervention Trial. Am J Cardiol. 1986;58:1-13.

8. Kannel WB, Dawber TR, Kagan A, Revotskie N, Stokes J 3rd. Factors of risk in the development of coronary heart disease - six year follow-up experience. The Framingham Study. Ann Intern Med. 1961;55:33-50.

9. Reaven GM. Banting lecture 1988. Role of insulin resistance in human disease. Diabetes. 1988;37:1595-607.

10. Alberti KG, Eckel RH, Grundy SM, et al. Harmonizing the metabolic syndrome: a joint interim statement of the International Diabetes Federation Task Force on Epidemiology and Prevention; National Heart, Lung, and Blood Institute; American Heart Association; World Heart Federation; International Atherosclerosis Society; and International Association for the Study of Obesity. Circulation. 2009;120:1640-5.

11. The Task Force on Diabetes and Cardiovascular Diseases of the European Society of Cardiology (ESC) and of the European Association for the Study of Diabetes (EASD). Guidelines on diabetes, pre-diabetes, and cardiovascular diseases: executive summary. Eur Heart J. 2007;28:88-136.
12. Conroy RM, Pyörälä K, Fitzgerald AP, et al. Estimation of tenyear risk of fatal cardiovascular disease in Europe: the SCORE project. Eur Heart J. 2003;24:987-1003.

13. Cifkova R, Byma S, Ceska R, et al. Prevention of cardiovascular diseases in adults. Task force of the Czech medical societies. Vnitr Lek. 2005;51(9):1021-36 (in Czech).

14. Kahn R, Buse J, Ferrannini E, Stern M. American diabetes association; European Association for the study of diabetes. The metabolic syndrome: time for a critical appraisal: joint statement from the American Diabetes Association and the European Association for the Study of Diabetes. Diab Care. 2005;28: 2289-304.

15. Vague J. The degree of masculine differentiation of obesities: a factor determining predisposition to diabetes, atherosclerosis, gout and uric calculous disease. Am J Clin Nutr. 1956;4:20-34.

16. Lemieux I, Pascot A, Couillard C, et al. Hypertriglyceridemic waist. A marker of the atherogenic metabolic triad (hyperinsulinemia, hyperapolipoprotein B, small dense LDL) in men. Circulation. 2000;102:179-84.

17. Lamarche B, Tchemof A, Mauriége P, et al. Fasting insulin and apolipoprotein B levels and low-density lipoprotein particle size as risk factors for ischemic heart disease. JAMA. 1998;279:1955-61.

18. Vasan RS, Larson MG, Leip EP, et al. Impact of high-normal blood pressure on the risk of cardiovascular disease. N Engl $\mathrm{J}$ Med. 2001;345:1291-7.

19. Cooper-DeHoff RM, Gong Y, Handberg EM, et al. Tight blood pressure control and cardiovascular outcomes among hypertensive patients with diabetes and coronary artery disease. JAMA. 2010;304:61-8.

20. Fruchart JC, Sacks FM, Hermans MP, et al. The residual risk reduction initiative: a call to action to reduce residual vascular risk in dyslipidemic patients. A condensed position paper by the Residual Risk Reduction Initiative (R3I). Diab Vasc Dis Res. 2008;5:319-35.

21. Austin MA, Breslow JL, Hennekens CH, Buring JE, Willett WC, Krauss RM. Low-density lipoprotein subclass pattern and risk of myocardial infarction. JAMA. 1988;260:1917-21.

22. Sacks FM, Expert Group on HDL Cholesterol. The role of highdensity lipoprotein (HDL) in the prevention and treatment of coronary heart disease: expert group recommendations. Am J Cardiol. 2002;90:139-43.

23. Sarwar N, Danesh J, Eiriksdottir G, et al. Triglycerides and the risk of coronary heart disease: 10158 incident cases among 262 525 participants in 29 Western prospective studies. Circulation. 2007;115:450-8.

24. Assmann G, Schulte H, Cullen P, Seedorf U. Assessing risk of myocardial infarction and stroke: new data from the Prospective Cardiovascular Münster (PROCAM) study. Eur J Clin Invest. 2007;37:925-32.

25. McLaughlin T, Abbasi F, Cheal K, Chu J, Lamendola C, Reaven G. Use of metabolic markers to identify overweight individuals who are insulin resistant. Ann Intern Med. 2003;139:802-9.

26. Dobiasova M. Atherogenic index of plasma [ $\log ($ triglycerides/ HDL-cholesterol)]: theoretical and practical implications. Clin Chem. 2004;50:1113-5.

27. Dobiasova M. AIP atherogenic index of plasma as a significant predictor of cardiovascular risk: from research to clinical practice. Vnitr Lék. 2006;52:64-71 (in Czech).

28. The ACCORD Study Group. Effects of combination lipid therapy in type 2 diabetes mellitus. N Engl J Med. 2010;362:1563-74.

29. Action to Control Cardiovascular Risk in Diabetes Study Group, Gerstein HC, Miller ME, Byington RP, et al. Effects of intensive glucose lowering in type 2 diabetes. N Engl J Med. 2008; 358:2545-59.

30. Balkau B, Hu G, Qiao Q, et al. DECODE Study Group, European Diabetes Epidemiology Group. Prediction of the risk of cardio- 
vascular mortality using a score that includes glucose as a risk factor. The DECODE Study. Diabetologia. 2004;47:2118-28.

31. Saaristo T, Peltonem M, Lindstrom J, et al. Cross-sectional evaluation of the Finnish Diabetes Risk Score: a tool to identify undetected type 2 diabetes, abnormal glucose tolerance, and metabolic syndrome. Diab Vasc Dis Res. 2005;2:67-72.

32. Rosolova H, Petrlova B, Simon J, Sifalda P, Sipova I. Highsensitivity $\mathrm{C}$-reactive protein and the hypertriglyceridemic waist in patients with type 2 diabetes and metabolic syndrome. Med Sci Monit. 2008;14:CR411-5.

33. The Emerging Risk Factors Collaboration. C-reactive protein concentration and risk of coronary heart disease, stroke, and mortality: an individual participant meta-analysis. Lancet. 2010; 375:132-40.

34. Haffner SM. The metabolic syndrome: inflammation, diabetes mellitus, and cardiovascular disease. Am J Cardiol. 2006;97:3A-11A.

35. Berl T, Henrich W. Kidney-heart interactions: epidemiology, pathogenesis and treatment. Clin J Am Soc Nephrol. 2006;1:8-18.

36. Patel A, ADVANCE Collaborative Group. Effects of a fixed combination of perindopril and indapamide on macrovascular and microvascular outcomes in patients with type 2 diabetes mellitus (the ADVANCE trial): a randomised controlled trial. Lancet. 2007;370:829-40.

37. The ADVANCE Collaborative Group. Intensive blood glucose control and vascular outcomes in patients with type 2 diabetes. $\mathrm{N}$ Engl J Med. 2008;358:2560-72.

38. Palazzuoli A, Gallotta M, Quatrini I, Nuti R. Natriuretic peptide (BNP, NT-proBNP) measurement and relevance in heart failure. Vasc Health Risk Manag. 2010;6:411-8.

39. Mayer O, Simon J, Plásková M, Cífková R, Trefil L. N-terminal pro-Brain natriuretic peptide as prognostic marker for mortality in coronary patients without clinically manifested heart failure. Eur J Epidemiol. 2009;24:363-8.

40. Olsen MH, Hansen TW, Christensen MK, et al. N-terminal probrain natriuretic peptide, but not high sensitivity $\mathrm{C}$-reactive protein, improves cardiovascular risk prediction in the general population. Eur Heart J. 2007;28:1374-81.

41. Sipilä K, Moilanen L, Nieminen T, et al. Metabolic syndrome and carotid intima media thickness in the Health 2000 survey. Atherosclerosis. 2009;204:276-81.
42. Allison MA, Wright CM. Age and gender are the strongest clinical correlates of prevalence of coronary calcification. Int $\mathrm{J}$ Cardiol. 2005;98:325-30.

43. Hunt ME, O'Malley PG, Feuerstein I, Taylor AJ. The relationship between the 'metabolic score' and sub-clinical atherosclerosis detected with electron beam computed tomography. Coron Artery Dis. 2003;14:317-22.

44. Meigs JB, Larson MG, D'Agostino RB, et al. Coronary artery calcification in type 2 diabetes and insulin resistance: the framingham offspring study. Diab Care. 2002;25:1313-9.

45. LaMonte MJ, FitzGerald SJ, Church TS, et al. Coronary artery calcium score and coronary heart disease events in a large cohort of asymptomatic men and women. Am J Epidemiol. 2005;162: 421-9.

46. Uretsky S, Rozanski A, Singh P et al. The presence, characterization and prognosis of coronary plaques among patients with zero coronary calcium scores. Int J Cardiovasc Imaging. 2010; doi:10.1007/s10554-010-9730-0.

47. Schmermund A, Achenbach S, Budde T, et al. Effect of intensive versus standard lipidlowering treatment with atorvastatin on the progression of calcified coronary atherosclerosis over 12 months: a multicenter, randomized, double-blind trial. Circulation. 2006;113:427-37.

48. McEvoy JW, Blaha MJ, Defilippis AP, et al. Coronary artery calcium progression: an important clinical measurement? A review of published reports. J Am Coll Cardiol. 2010;56:1613-22.

49. Ramakrishna G, Miller TD, Breen JF, Araoz PA, Hodge DO, Gibbons RJ. Relationship and prognostic value of coronary artery calcification by electron beam computed tomography to stressinduced ischemia by single photon emission computed tomography. Am Heart J. 2007;153:807-14.

50. Rasmussen-Torvik LJ, Li M, Kao W, et al. Association of a fasting glucose genetic risk score with subclinical atherosclerosis: The Atherosclerosis Risk in Communities (ARIC) Study. Diabetes. 2011;60:331-5.

51. Pechlivanis S, Scherag A, Mühleisen TW, et al. Heinz Nixdorf Recall Study Group. Coronary artery calcification and its relationship to validated genetic variants for diabetes mellitus assessed in the Heinz Nixdorf recall cohort. Arterioscler Thromb Vasc Biol. 2010;30:1867-72. 